\title{
DEBATE
}

\section{Persistent organic pollutants in food: public health implications}

\author{
E Fattore, R Fanelli, C La Vecchia
}

J Epidemiol Community Health 2002;56:831-832

Persistent organic pollutants are a heterogeneous group of chemicals sharing a number a common properties, including long term persistence and diffusion in the environment, and bioaccumulation through the food chain. Animal experiments and epidemiological studies suggest that the most sensitive adverse effects, such as disturbances on metabolism, development, and reproductive system, may occur in the range of current human exposure. Although the potential cancer risk of persistent organic pollutants remains undefined, the implementation of actions to reduce the exposure to these substances, which mainly occurs through the diet, is important.

See end of article for authors' affiliations

Correspondence to: Carlo La Vecchia, Istituto di Ricerche Farmacologiche "Mario Negri", Via Eritrea 62, 20157 Milano, Italy; bonifacino@marionegri.it
$\mathrm{P}$ ersistent organic pollutants (POPs) are a heterogeneous group of chemicals including organochlorine pesticides, industrial pollutants such as polychlorinated biphenyls (PCBs), and unintentional byproducts of chemical manufacturing and combustion processes, such as dioxins and furans. Although these groups of chemicals include hundreds of different compounds with different properties and toxic potencies, they share a number a common properties, including their long term persistence and diffusion in the environment, and their bioaccumulation through the food chain. For this reason it is not surprising that for the general population the main route of exposure is through the diet.

Generally speaking, these compounds are not considered so harmful for their acute toxicity (also because of their low concentrations in the environment) but for long term sub-lethal effects, such as reproductive, developmental and immunological disturbances, and for cancer. As summarised in table 2 of the paper by Schafer and Kegley, all these compounds show evidence of endocrine disruptor status in animals or humans. ${ }^{1}$ This means that POPs have the ability, in different ways, to disturb the normal hormonal balance in living organisms. At the fetal stage the sensitivity to these effects can be very high, resulting in developmental disorders persisting during the adult life. For TCDD (the most toxic congener of the dioxin group), for instance, prenatal exposure has been shown to result in adverse effects on the reproductive system, including a decrease of spermatogenesis. ${ }^{2}$ In humans an indication of the relation between exposure to TCDD and effects on reproduction and development arises from the changed sex ratio observed in the exposed population after the accidental release of TCDD in Seveso, Italy, in 1976. ${ }^{3}$ It is unclear, however, whether lower levels of exposure can introduce any appreciable changes in sex ratios. ${ }^{4}$ The more disquieting epidemiological investigations may be those on the effects in children after prenatal exposure at "background" levels of dioxins and PCBs. In a cohort of Dutch children, for example, in uterus exposure to these chemicals has been associated with poorer cognitive functioning. ${ }^{5}$

The potential implications of POPs on cancer risk are unclear and substantially different between different POPs. As shown in table 2 of the above mentioned paper, ${ }^{1}$ two of these substances have been classified by the International Agency for Research on Cancer (IARC) as known human carcinogens. These are the TCDD again, and the 2,3,4,7,8pentachlorodibenzuran, the most toxic congener of the furan group. TCDD is well known as a multiorgan carcinogen in animals; the target organs include liver, thyroid, lung, skin, and soft tissues. A substantial body of evidences indicates that the mode of action of carcinogenicity - as well as of non-cancer effects-of TCDD involves the binding to a cytoplasmatic protein receptor, $A h$. Once bound to the ligand, the $A h$ receptor would reach the cell nucleus, binding to a specific DNA domain, and this would enhance the transcription of target genes involved in several processes, including cell growth and differentiation. These findings are consistent with the hypothesis that TCDD exerts its toxic action as promoter rather than as initiator in the process of carcinogenesis. As there is a general agreement that other POPs, the so called "dioxin-like" compounds, share the same mode of action through the Ah receptor it is unlikely that they are complete human carcinogens too. More generally, there is a lack of epidemiological evidence of the carcinogenicity of most POPs.

The occupational or massive accidental exposure to TCDD has been associated to an excess of incidence of soft tissue sarcomas and perhaps non-Hodgkin's lymphomas. ${ }^{67}$ None the less in these cases the levels of exposure were much higher than the ones commonly reached through the diet. The question of to what extent the current environmental exposure to dioxins and other "dioxin- like" compounds is linked to an increase of cancer risk in the general population is still without a clear answer, and even less is

Abbreviations: POPs, persistent organic pollutants; PCBs, polychlorinated biphenyls 
known about the other POPs. For instance, investigations focused on fishermen populations in the east coast of Sweden, more exposed than the general population to POPs through fat fish consumption, have not shown an increased cancer risk. Furthermore, the attempts to establish a more direct link between the risk of cancer and the background levels of POPs is made complex by the observation that over the past two decades most common digestive tract cancers substantially declined in developed countries (stomach and more recently colorectum $)^{8}$ as well as in developing ones (stomach); breast cancer mortality has also been downwards over recent years. ${ }^{9}$ On the other hand, recent trends in mortality are inconsistent and difficult to interpret for other cancers, such as liver cancer and soft tissue sarcomas, while trends are generally upward for non-Hodgkin's lymphomas, ${ }^{10}$ which are considered to be potential target of POPs. Thus, while for cancer it is very difficult to assess if and at which concentrations POPs constitute a human health risk, animal experiments and epidemiological studies suggest that the most sensitive adverse effects, such as disturbances on metabolism, development, and reproductive system, may occur in the range of current human exposure. The implementation of actions to reduce the exposure to these substances, which mainly occurs through the diet, is important, in particular to protect the most vulnerable subjects (for example, pregnant women, nursing infants, subjects living near local sources, and others), if and where these can be identified.

\section{ACKNOWLEDGEMENTS}

Work conducted with contribution from the Italian Association for Cancer Research, Milan. The authors thank Mrs M P Bonifacino for editorial editing.

\section{Authors' affiliations}

E Fattore, R Fanelli, Istituto di Ricerche Farmacologiche "Mario Negri", Milan, Italy

C La Vecchia, Istituto di Statistica Medica e Biometria, Università degli Studi di Milano, Milan, Italy

\section{REFERENCES}

1 Schafer KS, Kegley SE. Persistent toxic chemicals in the US food supply. J Epidemiol Community Health 2002;56:813-17.

2 Mably TA, Bjerke DL, Moore RW, et al. In utero and lactational exposure of male rats to 2,3,7,8-tetrachlorodibenzo-p-dioxin. 3. Effects on the spermatogenesis and reproductive capability. Toxicol Appl Pharmacol 1992;114:118-26.

3 Moccarelli P, Gerthoux PM, Ferrari E, et al. Paternal concentrations of dioxin and sex ratio of offspring. Lancet 2000;355: 1858-63.

4 Parazzini F, La Vecchia C, Levi F, et al. Trends in male:female ratio among newborn infants in 29 countries from five continents. Hum Reprod 1998;13:1394-6.

5 Patandin S, Lanting $\mathrm{Cl}$, Mulder PG, et al. Effects of environmental exposure to polychlorinated biphenyls and dioxins on cognitive abilities in Dutch children at 42 months of age. J Pediatr 1999;134:33-41.

6 Kogevinas $M$, Becher $\mathrm{H}$, Benn $T$, et al. Cancer mortality in workers exposed to phenoxy herbicides, chlorophenols, and dioxins. An expanded and updated international cohort study. Am J Epidemiol 1997; 145: 1061-75.

7 Bertazzi PA, Consonni D, Bachetti S, et al. Health effects of dioxin exposure: a 20-year mortality study. Am J Epidemiol 2001;153:1031-

8 Fernandez E, Bosetti C, La Vecchia C, et al. Sex differences in colorectal cancer mortality in Europe, 1955-1996. Eur J Cancer Prev 2000:9:99-104.

9 Levi $\mathbf{F}$, Lucchini $F$, Negri $E$, et al. The fall in breast cancer mortality in Europe. Eur J Cancer 2001;37:1409-12.

10 Levi F, Lucchini F, Negri E, et al. Trends in mortality from non-Hodgkin's lymphomas. Leuk Res 2002;518:1-6 